\title{
Lentivirus-mediated shRNA targeting XIAP and survivin inhibit SW1990 pancreatic cancer cell proliferation in vitro and in vivo
}

\author{
CHUN JIANG ${ }^{1}$, TAN TAN $^{3}$, XIAO-PING YI ${ }^{1}$, HONG SHEN $^{2}$ and YI-XIONG LI ${ }^{1}$ \\ ${ }^{1}$ Department of General Surgery, and ${ }^{2}$ Medical Research Center, Xiangya Hospital, Central South University, Hunan 410008; \\ ${ }^{3}$ Department of Clinical Laboratory, The First People's Hospital of Chenzhou, Hunan 423000, P.R. China
}

Received December 8, 2010; Accepted April 4, 2011

DOI: 10.3892/mmr.2011.472

\begin{abstract}
The aim of this study was to investigate the inhibitor of apoptosis proteins survivin and XIAP in pancreatic cancer by determining their biological characteristics and expression. XIAP and survivin are potential therapeutic targets for pancreatic cancer, and elucidating their association with cell proliferation and apoptosis may lead to the development of novel treatments for this disease. The human pancreatic cancer SW1990 cell line was infected with lentivirus and then analyzed by real-time PCR, and the results were confirmed by Western blotting. The MTT assay and the determination of caspase-3/-7 activity, DAPI-staining and tumorigenicity were used to measure cell proliferation and apoptosis in the human pancreatic cancer SW1990 cell line and in an experimental pancreatic cancer mouse xenograft model inoculated with the lentivirus-transfected SW1990 cells. The results revealed that the XIAP and survivin proteins were differentially expressed among the pancreatic cancer cell lines, and their decreased expression resulted in the inhibition of cell proliferation in vitro as well as in vivo. These findings suggest that lentivirus-mediated gene therapy targeting XIAP and survivin is a potential and attractive strategy for the treatment of pancreatic cancer.
\end{abstract}

\section{Introduction}

Pancreatic cancer is one the most common types of cancer and the fourth leading cause of mortality in the United States. Due to its low resectability and resistance to chemoradiotherapy, it is also one of the most aggressive human malignancies, with an extremely poor 5-year survival rate (as low as 3-4\%) (1). Pancreatic cancer results from deregulated cell proliferation, which constitutes the minimal common platform upon which all neoplastic evolution occurs. Therefore, adroit targeting of this critical event in cell proliferation may have potent and specific therapeutic consequences (2).

Correspondence to: Dr Yi-Xiong Li, Department of General Surgery, Xiangya Hospital, Central South University, Changsha, Hunan 410008, P.R. China

E-mail: liyixiong6@hotmail.com

Key words: XIAP, survivin, pancreatic cancer, proliferation, apoptosis
XIAP and survivin are two important members of the inhibitor of apoptosis (IAP) gene family, and were first identified as baculoviral proteins capable of inhibiting apoptosis. The Drosophila homolog of XIAP has been found to play a key role in cell proliferation, with loss of function resulting in embryonic cell death (3). Survivin promotes cell proliferation via its binding to microtubules, and assists in chromosomal segregation and cytokinesis during mitosis (4). XIAP and survivin contribute to the biological properties of cell survival and proliferation in ameloblastomas (5), and have been found to be up-regulated in numerous types of malignancies (6). In cancer patients, high levels of XIAP or survivin in tumor tissue have been reported to be associated with decreased overall survival, increased recurrence and resistance to chemotherapy (7). Furthermore, XIAP and survivin are metastasis genes, and are thus potential targets for the control of tumor growth and angiogenesis. Intermolecular cooperation between XIAP and survivin stimulates tumor cell invasion and promotes metastasis (8). It has been demonstrated that their inhibition by siRNA in melanoma inhibits tumor proliferation and promotes the sensitivity of the cell lines to apoptosis induced by chemotherapy (9). However, the effects of XIAP and survivin knockdown on the proliferation and apoptosis of pancreatic cancer cells remain to be investigated.

In the present study, the human XIAP and survivin genes were supressed by shRNA lentivirus vectors in the SW1990 human pancreatic cell line, which is derived from samples of splenic metastases (10) and exhibits the most suitable characteristics for XIAP and survivin study, as confirmed by a comparison of the expression status of IAPs among four different pancreatic cancer cell lines. In addition to examining the effects of shRNA lentivirus targeting human XIAP and/or survivin on the proliferation and apoptosis of SW1990 cells in vitro, the effects were examined in an in vivo experimental pancreatic cancer mouse xenograft model inoculated with lentivirus-transfected SW1990 cells.

\section{Materials and methods}

Construction of short hairpin RNA (shRNA) lentivirus targeting human XIAP and survivin. Three putative candidate shRNA sequences targeting human XIAP (GenBank accession no: NM 001167), survivin (GenBank accession no: NM 001168) and two negative control lentivirus vectors with a scrambled non-targeting shRNA sequence (Table I) were 
Table I. Target sequences of RNAi against human XIAP (NM 001167) and survivin (NM 001168).

\begin{tabular}{|c|c|c|}
\hline Vector & Target sequences & Sequences cloned into the vector \\
\hline Lv-X1 & ggtgaaggtgataaagtaa & $\begin{array}{l}\text { F: 5'-ccggtaggtgaaggtgataaagtaattcaagagattactttatcaccttcacctatttttg-3' } \\
\text { R: 5'-aattcaaaaataggtgaaggtgataaagtaatctcttgaattactttatcaccttcaccta-3' }\end{array}$ \\
\hline $\mathrm{Lv}-\mathrm{X} 2$ & cttgaggagtgtctggtaa & $\begin{array}{l}\text { F: 5'-ccggcacttgaggagtgtctggtaattcaagagattaccagacactcctcaagtgtttttg-3' } \\
\text { R: 5'-aattcaaaaacacttgaggagtgtctggtaatctcttgaattaccagacactcctcaagtg-3' }\end{array}$ \\
\hline Lv-X3 & gtggtagtcctgtttcagc & $\begin{array}{l}\text { F: 5'-ccggaagtggtagtcctgtttcagcttcaagagagctgaaacaggactaccactttttttg-3' } \\
\text { R: 5'-aattcaaaaaaagtggtagtcctgtttcagctctcttgaagctgaaacaggactaccactt-3' }\end{array}$ \\
\hline Lv-Xnc & ttctccgaacgtgtcacgt & $\begin{array}{l}\text { F: 5'-ccggttctccgaacgtgtcacgtttcaagagaacgtgacacgttcggagaatttttg-3' } \\
\text { R: 5'-aattcaaaaattctccgaacgtgtcacgttctcttgaaacgtgacacgttcggagaa-3' }\end{array}$ \\
\hline Lv-S1 & gaggctggcttcatccactgcc & $\begin{array}{l}\text { F: 5'-ccgggaggctggcttcatccactgcttcaagagagcagtggatgaagccagcctctttttg-3' } \\
\text { R: 5'-aattcaaaaagaggctggcttcatccactgctctcttgaagcagtggatgaagccagcctc-3' }\end{array}$ \\
\hline Lv-S2 & aaggaccaccgcatctctacat & $\begin{array}{l}\text { F: 5'-ccggaaggaccaccgcatctctacattcaagagatgtagagatgcggtggtccttttttg-3' } \\
\text { R: 5'-aattcaaaaaaaggaccaccgcatctctacatctcttgaatgtagagatgcggtggtcctt-3' }\end{array}$ \\
\hline Lv-S3 & gagaaagtgcgccgtgccatcg & $\begin{array}{l}\text { F: 5'-ccgggagaaagtgcgccgtgccatcttcaagagagatggcacggcgcactttctctttttg-3' } \\
\text { R: 5'-aattcaaaaagagaaagtgcgcegtgccatctctcttgaagatggcacggegcactttctc-3' }\end{array}$ \\
\hline Lv-Snc & ttctccgaacgtgtcacgt & $\begin{array}{l}\text { F: 5'-ccggttctccgaacgtgtcacgtttcaagagaacgtgacacgttcggagaatttttg-3' } \\
\text { R: 5'-aattcaaaaattctccgaacgtgtcacgttctcttgaaacgtgacacgttcggagaa-3' }\end{array}$ \\
\hline
\end{tabular}

synthesized by Genechem (Shanghai, China) and cloned into two self-inactivating lentivirus vectors: pGCSIL-PUR (Genechem), containing a CMV-driven Puromycin for XIAP shRNA, and pGCSIL-NEO (Genechem), containing a CMV-driven neomycin for survivin shRNA. The synthesized oligonucleotides were annealed and inserted at the cloning restriction sites of $A g e \mathrm{I}$ and $E c o$ RI. The positive clones were identified by restriction mapping and DNA sequencing. The lentiviral vectors with a positive insert and the packaging vectors (pHelper1.0 and 2.0) were then transfected into 293T cells using Lipofectamine ${ }^{\mathrm{TM}}$ reagent (Invitrogen, Carlsbad, CA, USA). The supernatant containing the lentiviral particles was collected every $12 \mathrm{~h}$ from 24 until $72 \mathrm{~h}$ post-transfection and filtered through a $0.45-\mu \mathrm{m}$ cellulose acetate filter. The final virus titer was $\sim 10^{9} \mathrm{TU} / \mathrm{ml}$, as determined by hole-bydilution titer assay.

Cell culture and lentiviral infection. The human pancreatic cancer cell lines SW1990, Panc-1, Mia-paca2 and BxPc-3 were cultured in DMEM supplemented with $10 \%$ fetal bovine serum, $100 \mu \mathrm{g} / \mathrm{ml}$ streptomycin and $100 \mathrm{U} / \mathrm{ml}$ penicillin at $37^{\circ} \mathrm{C}$ in a humidified atmosphere of $95 \%$ air and $5 \% \mathrm{CO}_{2}$. Cells reaching $30-50 \%$ confluence were infected with viral supernatants at a multiplicity of infection (MOI) of 50 in the presence of polybrene at a final concentration of $5 \mu \mathrm{g} /$ $\mathrm{ml}$, then cultured in ENi.S (Genechem) for $8 \mathrm{~h}$ followed by further culture in DMEM medium containing $0.5 \mu \mathrm{g} /$ $\mathrm{ml}$ puromycin (Sigma-Aldrich, St. Louis, MO, USA) and/ or $400 \mu \mathrm{g} / \mathrm{ml}$ neomycin (Sigma-Aldrich) for the selection of positive lentiviral-transfected cells. To achieve the efficient and simultaneous inhibition of both the XIAP and survivin genes, the lentiviruses were sequentially transfected into the SW1990 cells in order of highest inhibition efficiency for XIAP and survivin. In brief, the pancreatic cancer cells were first transfected with XIAP shRNA lentivirus and selected by puromycin, then the positive cell clones with stable XIAP shRNA transfection were transfected with survivin shRNA lentivirus and selected by neomycin.

$R N A$ extraction and real-time RT-PCR quantitation. Total RNA was isolated from pancreatic cancer cells by TRIzol reagent (Invitrogen) according to the manufacturer's instructions. First strand cDNA synthesis was performed using the Advantage RT-for-PCR kit (Clontech Laboratories Inc., Palo Alto, CA, USA) as previously described. The specific primers for XIAP, survivin and $\beta$-actin were designed from their GenBank sequences and synthesized by Bio Basic Inc. (Shanghai, China) (Table II). Real-time PCR quantitation for individual target mRNA expression was performed on an ABI Model 7500 Sequence Detector (Applied Biosystems, Foster City, CA, USA) using a real-time PCR kit (Takara Biotechnology, Otsu, Japan). The reaction parameters were $95^{\circ} \mathrm{C}$ for $30 \mathrm{sec}$, followed by 40 cycles at $95^{\circ} \mathrm{C}$ for $5 \mathrm{sec}$ and $60^{\circ} \mathrm{C}$ for $30 \mathrm{sec}$. The amplified PCR products were quantified by measuring the target and $\beta$-actin mRNA calculated cycle thresholds (CT). The amount of specific mRNA in each sample was calculated from the standard curve and normalized to the $\beta$-actin mRNA. The comparative $2^{-\Delta \Delta \mathrm{CT}}$ method was used for relative quantification and statistical analysis. The results were expressed as the $\mathrm{n}$-fold difference relative to blank vector transfection controls.

Western blot analysis. Total protein was lysed in RIPA buffer containing $50 \mathrm{mM}$ Tris- $\mathrm{HCl}(\mathrm{pH} 7.3), 150 \mathrm{mM} \mathrm{NaCl}$, $2 \%$ NP-40, $0.5 \%$ deoxycholate, $2 \mathrm{mM}$ EDTA, $2 \mathrm{mM} \mathrm{NaF}$ and $1 \%$ Protease Inhibitor Cocktail (Pierce, Rockford, IL, USA). Protein concentrations were assessed by the Bradford assay (Bio-Rad Laboratories Inc., Hercules, CA, USA). Protein $(20 \mu \mathrm{g})$ from the cell lysates was separated on $12 \%$ sodium dodecylsulfate-polyacrylamide gel under reducing conditions, 
Table II. Nucleotide sequences of primers for real-time PCR.

\begin{tabular}{ll}
\hline Gene & \multicolumn{1}{c}{ Primer sequences } \\
\hline XIAP & F: 5'-gacagtatgcaagatgagtcaagtca-3' \\
& R: 5'-gcaaagcttctcctcttgcag-3' \\
Survivin & F: 5'-aggtcatctcggctgttcctg-3' \\
& R: 5'-tcatcctcactgcggctgtc-3' \\
$\beta$-actin & F: 5'-actcttccagcttccttcc-3' \\
& R: 5'-gtacttgcgctcaggaggag-3'
\end{tabular}

then transferred onto Nitroplus-2000 membranes (Micron Separations Inc., Westborough, MA, USA). Equal loading of each lane was monitored by Ponceau-S staining. Non-specific antibody binding was blocked by pre-incubation in $1 \mathrm{X}$ Trisbuffered saline (TBS) containing 5\% skim milk for $1 \mathrm{~h}$ at room temperature. The membranes were then incubated overnight at $4^{\circ} \mathrm{C}$ with goat anti-human XIAP antibody (R\&D Systems Inc., Minneapolis, MN, USA) at a 1:5,000 dilution ratio and rabbit anti-human survivin antibody (Novus Biologicals, Inc.) at a 1:2,000 dilution ratio in $1 \mathrm{X}$ TBST containing 3\% skim milk. After washing, the membranes were incubated with HRP-conjugated secondary antibodies (Sigma-Aldrich) at a $1: 10,000$ dilution ratio for $1 \mathrm{~h}$ at room temperature. Bands were visualized by employing the ECL kit according to the manufacturer's instructions.

MTT assay. To observe the effects of XIAP and/or survivin on the in vitro proliferation of the pancreatic cancer cells, SW1990 cells in the logarithmic phase of growth were seeded at $1.0 \times 10^{3}$ cells/well in 96-well microplates (Nunc, Denmark) overnight, and were then respectively transfected with recombinant lentivirus. Cell growth was examined by the MTT assay after the lentiviral transfection once a day for 8 days. Briefly, $20 \mu 1$ of MTT solution $(5 \mathrm{mg} / \mathrm{ml}$ in PBS) was added to each well followed by incubation for $4 \mathrm{~h}$ at $37^{\circ} \mathrm{C}$. The cells were lysed in $100 \mu 1$ lysis buffer and analyzed on a ThermoMax microplate reader (Bio-Rad Laboratories Inc.) at a wavelength of $490 \mathrm{~nm}$. The experiments were independently repeated in triplicate.

Tumorigenicity experiments. The effects of XIAP and survivin silencing on tumor growth were additionally examined in vivo in an experimental pancreatic cancer xenograft model. A suspension of SW1990 cells $\left(1.5 \times 10^{7}\right.$ cells in $200 \mu$ l DMEM) in the logarithmic phase of growth was subcutaneously injected into the left axilla of 6-week-old nu/nu BALB/c mice from the Central Animal Care Unit of Central South University, China. The mice were maintained under a 12-h light/dark cycle with ad libitum access to food and water. The animals received humane care in compliance with the university's ethical guidelines. After inoculation, the tumors were measured weekly in two perpendicular diameters using a caliper, and the tumor volume was determined using the following formula: $V=\pi / 6 x$ [large diameter $\mathrm{x}$ (short diameter) ${ }^{2}$ ]. The mice were randomly divided into 7 groups according to which lentivirus was transfected into the cells: the Lv-Xnc, Lv-Snc, Lv-Xnc+Snc groups, their respective corresponding control groups, as well as a blank control group, which had no recombinant lentiviral transfection.

Caspase-3/-7 activity. Caspase-3/-7 activity was evaluated using the Caspase-Glo ${ }^{\circledR}-3 /-7$ assay (Promega, Madison, WI, USA) according to the manufacturer's instructions. The cells were seeded onto a 6 -well plate at a density of $1 \times 10^{5}$ cells/well and incubated at $37^{\circ} \mathrm{C}$ for $72 \mathrm{~h}$. Luminescence was detected on a Sirius luminometer (version 3.2; Berthold Inc., Germany) with a $3 \mathrm{sec}$ delay time and $10 \mathrm{sec}$ measurement time. The caspase$3 /-7$ activity was normalized to the number of viable cells as determined by trypan blue staining, and the caspase-3/-7 fold induction by XIAP and/or survivin shRNA lentivirus was determined as the ratio of caspase-3/-7 activity between the treated and control groups.

DAPI staining and calculation of SW1990 apoptosis index. The cells were seeded at a density of $2 \times 10^{4}$ cells/well on sterile round glass cover-slips placed in 24-well plates, then cultured for $72 \mathrm{~h}$. After being rinsed twice with phosphatebuffered saline (PBS), the cells were fixed in PBS containing $4 \%$ paraformaldehyde and $10 \%$ sucrose at room temperature for $15 \mathrm{~min}$. The cell nuclei were then labeled with $1 \mu \mathrm{g}$ / $\mathrm{ml}$ DAPI (Sigma-Aldrich) at room temperature for $2 \mathrm{~min}$ in the dark, mounted with glycergel solution $(60 \%, 4 \mu \mathrm{l})$ (SouthernBiotech, Birmingham, AL, USA) and visualized under a fluorescence microscope. The apoptotic cells were observed by the characteristics of condensing, shrinking and rupturing nuclei, and were counted in three randomized microscopic fields. The apoptosis index (AI) of the cultured SW1990 cells with different lentiviral transfections was calculated using the following formula: AI $(\%)=$ apoptotic cells/total cells $\mathrm{x} 100 \%$.

Statistical analysis. Data are expressed as the means \pm SD. Analysis was performed using ANOVA or the Student's t-test. All statistical analyses were performed using SPSS 13.0 software (version 11.5; SPSS Inc., Chicago, IL, USA). P<0.05 was considered statistically significant.

\section{Results}

Real-time RT-PCR and Western blot quantification in pancreatic cancer cell lines. To better determine the relative mRNA and protein expression levels of XIAP and survivin using a calibrator in the pancreatic cancer cell lines, the mRNA expression level of Bxpc-3 cells was set at a value of 1 since, of the four cell lines, it exhibited the lowest expression levels of the genes of interest. Real-time RT-PCR demonstrated that the relative mRNA expression levels of the SW1990, Panc-1 and Mia-paca2 cells were 1.98 $\pm 0.28-, 3.03 \pm 0.23$ - and 1.24 \pm 0.06 fold for XIAP, and 3.95 $\pm 0.40-, 3.56 \pm 0.27$ - and 5.46 \pm 0.42 -fold for survivin, respectively (Fig. 1A). As indicated by Western blot gels, in the SW1990, Panc-1 and Mia-paca2 cells the protein expression of XIAP and survivin was consistent with their mRNA expression (Fig. 1B). Considering the relative XIAP and survivin expression levels of each of the pancreatic cancer lines, particularly in terms of their tumor formation capacity in nude mice, the SW1990 cell line was selected for subsequent experiments. 
A

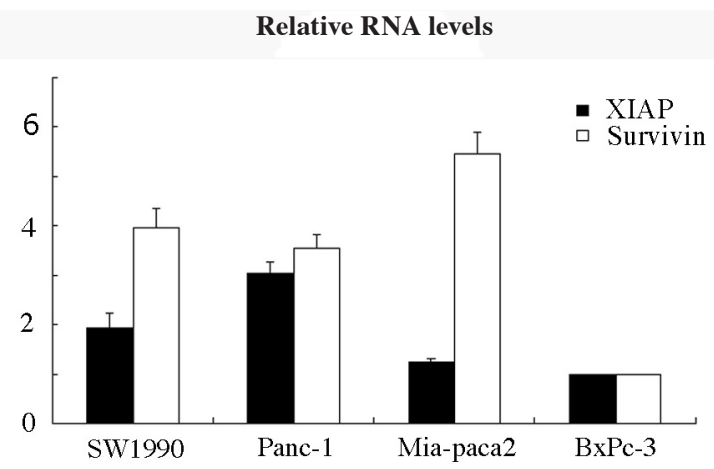

B

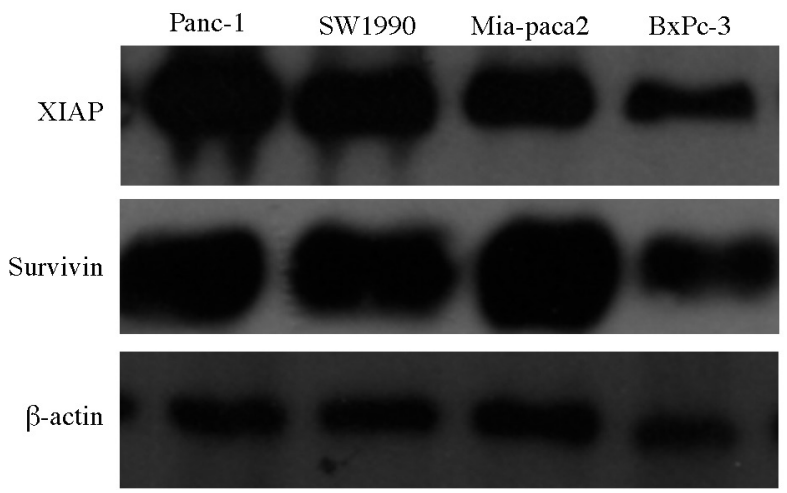

Figure 1. mRNA and protein expression of XIAP and survivin in the SW1990, Panc-1, Mia-paca2 and BxPc-3 pancreatic cancer cell lines. The expression level of XIAP and survivin in the Bxpc-3 cells was set as the calibrator with a relative expression value of 1 . $\beta$-actin was used as the internal loading control. The experiment was repeated three times independently.

Selection of the most effective XIAP and survivin-specific shRNA vector. Three different pairs of XIAP- and survivintargeting sequences were respectively designed and employed for the selection of the most effective shRNA lentivirus with the highest tranfection efficiency for the genes of interest. Compared to that in the SW1990 cells transfected with the Lv-Xnc blank vector, XIAP mRNA expression in the cells transfected with $\mathrm{Lv}-\mathrm{X} 1, \mathrm{Lv}-\mathrm{X} 2$ and $\mathrm{Lv}-\mathrm{X} 3$ was inhibited by $91.24,74.35$ and $71.43 \%$, respectively $(\mathrm{p}<0.05)$. Compared to that in the Lv-Snc group, survivin mRNA expression in the Lv-S1, Lv-S2 and Lv-S3 transfection groups was inhibited by $74.52,18.1$ and $9.27 \%$, respectively $(\mathrm{p}<0.05)$ (Fig. 2A). Western blotting revealed that the inhibition efficiencies on $\mathrm{XIAP}$ and survivin protein expression by the $\mathrm{Lv}-\mathrm{X} 1, \mathrm{Lv}-\mathrm{X} 2$, $\mathrm{Lv}-\mathrm{X} 3, \mathrm{Lv}-\mathrm{S} 1, \mathrm{Lv}-\mathrm{S} 2$ and $\mathrm{Lv}-\mathrm{S} 3$ lentiviruses were consistent with those on the mRNA expression of the targeted genes. Western gels were used to validate the specificity of the shRNA lentivirus targeting the XIAP or survivin genes. When either XIAP or survivin was knocked down, the expression level of the other IAP family members in the SW1990 cells was not affected by the transfected lentivirus (Fig. 2B and C). In addition, no difference was found in terms of the expression levels of XIAP or survivin between the nonsense vector-transfected SW1990 cells ( $\mathrm{Lv}$-Xnc or Lv-Snc) and the control SW1990 cells without lentiviral transfection ( $p>0.05)$ (Fig. 2). As a result, $\mathrm{Lv}-\mathrm{X} 1$ and $\mathrm{Lv}-\mathrm{S} 1$ were identified as the most effective recombinant lentiviruses and were used in subsequent experiments.
A Relative XIAP and survivin expression

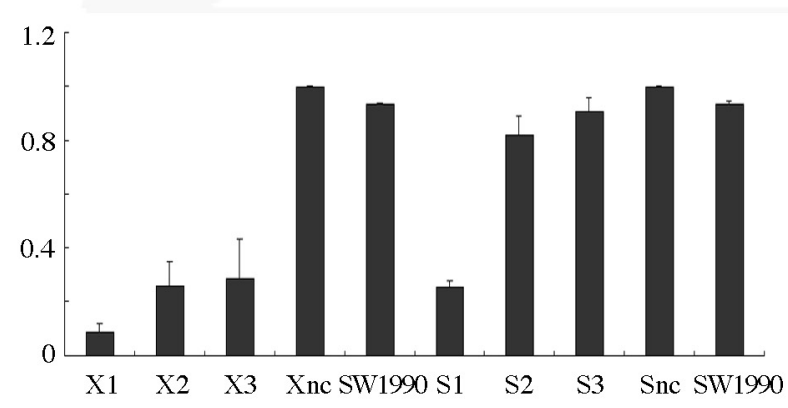

B

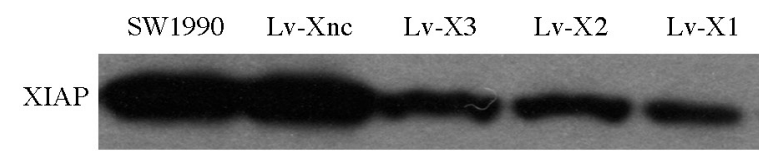

Survivin

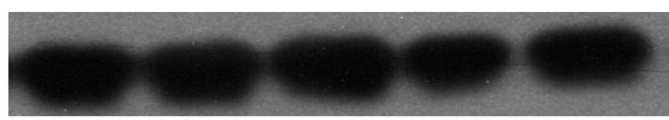

$\beta$-actin

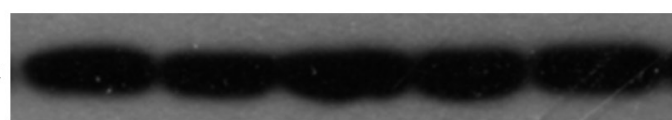

C

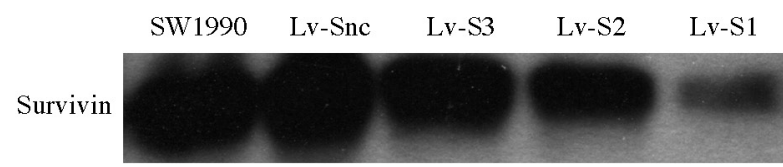

XIAP

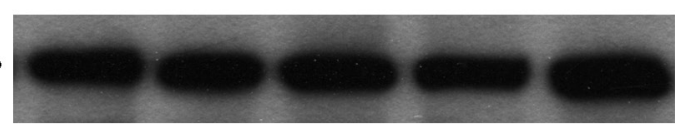

$\beta$-actin

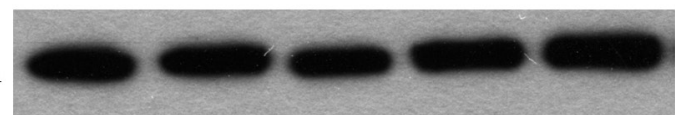

Figure 2. Inhibitory effects of three recombinant lentiviruses on the mRNA and protein expression of XIAP and survivin in SW1990 cells. The expression levels of XIAP and survivin in the Lv-Xnc and Lv-Snc groups, which were transfected with the nonsense lentivirus vector, was set as the calibrator with a relative expression value of 1 . $\beta$-actin was used as the internal loading control. The experiment was repeated three times independently.

Lentivirus-mediated simultaneous knockdown of XIAP and survivin in SW1990 cells. For a convenient evaluation of the transfection efficiency of shRNA lentivirus co-transfection, mRNA expression levels of XIAP and survivin in the control SW1990 cells, which were sequentially co-transfected with the blank vectors (nonsense insert lentivirus) of $\mathrm{Lv}$-Xnc and $\mathrm{Lv}-\mathrm{Snc}(\mathrm{Lv}-\mathrm{Xnc}+\mathrm{Snc})$, were set as the calibrator with a relative expression value of 1 . Real-time RT-PCR revealed no significant difference in XIAP and survivin expression between the two blank vector transfection control groups and the non-transfected SW1990 cells (XIAP, $0.85 \pm 0.19$; survivin, $0.92 \pm 0.12$ ). However, XIAP and survivin mRNA levels in the shRNA co-transfection group $(\mathrm{Lv}-\mathrm{X} 1+\mathrm{S} 1)$ were respectively inhibited by $0.47 \pm 0.02$ - and $0.52 \pm 0.03$-fold compared to the 
A

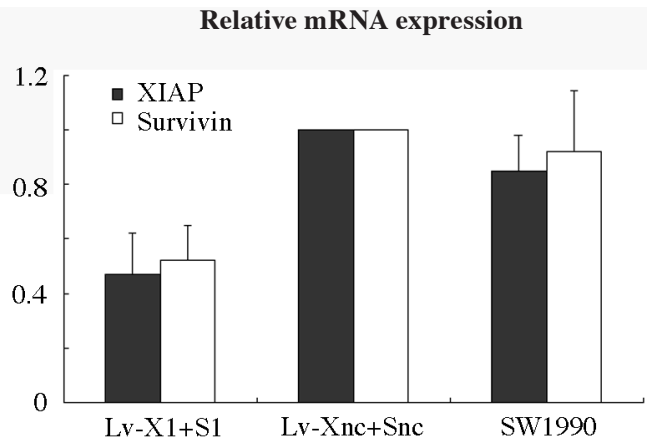

B

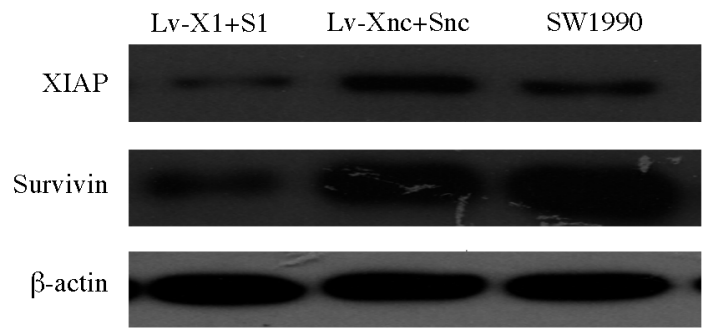

Figure 3. Simultaneous inhibition of XIAP and survivin by shRNA lentivirus co-transfection in SW1990 cells. Lv-X1+S1, experimental cells co-transfected with XIAP and survivin shRNA lentivirus; Lv-Xnc+Snc, control cells sequentially co-transfected with Lv-Xnc and Lv-Snc blank vectors; SW1990, blank control group in which the SW1990 cells were not transfected with any vector. $\beta$-actin was used as the internal loading control. The experiment was repeated three times independently.

Lv-Xnc+Snc control group (Fig. 3A). Recombinant shRNA lentivirus co-transfection significantly inhibited the mRNA expression of both XIAP and survivin $(\mathrm{p}<0.05)$. Consistent with the results of real-time RT-PCR, Western blotting confirmed the significant inhibition of the expression of the targeted genes in the $\mathrm{Lv}-\mathrm{X} 1+\mathrm{S} 1$ group at the protein level (Fig. 3B).

Inhibition of XIAP and survivin suppressed SW1990 cell proliferation in vitro and in vivo. At each daily check point from day 1 to day 8 following lentivirus transfection, there was no significant difference in the number of SW1990 cells among the four control groups, including the Lv-Xnc and Lv-Snc single blank vector transfection groups, sequential $\mathrm{Lv}-\mathrm{Xnc}$ and Lv-Snc co-transfection group ( $\mathrm{Lv}-\mathrm{Xnc}+\mathrm{Snc})$, and the untransfected SW1990 cell group ( $>0.05)$. However, single recombinant XIAP or survivin shRNA lentiviral transfection as well as simultaneous knockdown of XIAP and survivin significantly inhibited the proliferation of cultured SW1990 cells in vitro at the indicated time points from day 4 to day 8 $(\mathrm{p}<0.05)$. For example, the $\mathrm{OD}_{490}$ absorbance readings on day 8 in the untransfected, $\mathrm{Lv}-\mathrm{Xnc}, \mathrm{Lv}-\mathrm{Snc}$ and $\mathrm{Lv}-\mathrm{Xnc}+\mathrm{Snc}$ control groups and in the $\mathrm{Lv}-\mathrm{X} 1, \mathrm{Lv}-\mathrm{S} 1$ and $\mathrm{Lv}-\mathrm{X} 1+\mathrm{S} 1$ experimental groups were $1.990 \pm 0.003,1.876 \pm 0.004,1.873 \pm 0.003$ and $1.879 \pm 0.004$, and $0.879 \pm 0.004,0.732 \pm 0.004$ and $0.476 \pm 0.003$, respectively (Fig. 4A).

From day 16 to day 28 after SW1990 implantation, the tumorigenicity experiments revealed that both individual suppression of XIAP in the Lv-X1 group or survivin in the Lv-S1 group, as well as their combined knockdown in the $\mathrm{Lv}-\mathrm{X} 1+\mathrm{S} 1$ group, significantly inhibited the growth of the
A

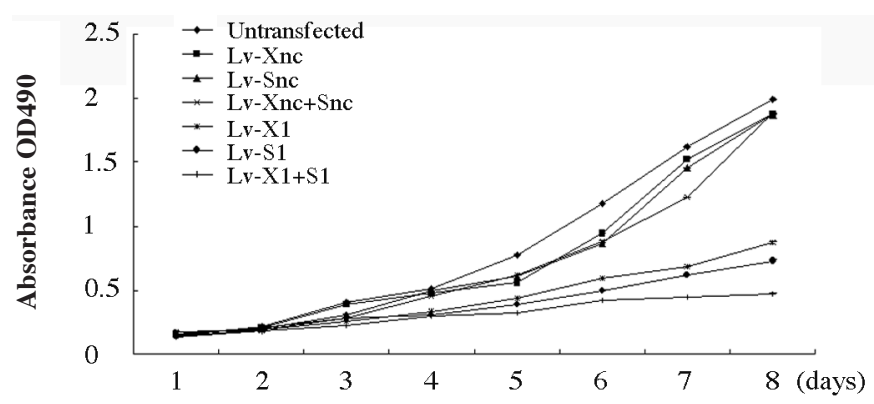

B

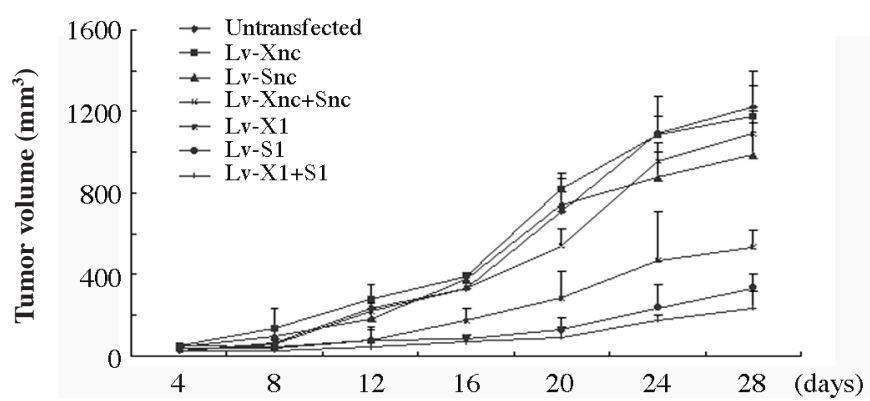

Figure 4. Suppression of SW1990 cell growth in vitro and tumorigenicity in vivo caused by inhibition of XIAP and/or survivin. (A) Cell growth curves of SW1990 cells transfected with XIAP and/or survivin shRNA lentivirus. (B) Tumor volume of xenografts in nude mice with suppressed XIAP and/or survivin expression following the inoculation of SW1990 cells. Untransfected, SW1990 cells without any transfection; Lv-X1, XIAP shRNA transfection group; Lv-S1, survivin shRNA transfection group; Lv-X1+S1, XIAP and survivin shRNA transfection group. Lv-Xnc, Lv-Snc and $\mathrm{Lv}-\mathrm{Xnc}+\mathrm{Snc}$ respectively represent the transfection control groups with nonsense inserts in the vectors corresponding to the above shRNA lentivirus.

transplanted tumors in the nude mice $(\mathrm{p}<0.05)$. Compared to the individual suppression in the Lv-X1 and Lv-S1 groups, the combined knockdown of XIAP and survivin in the $\mathrm{Lv}-\mathrm{X} 1+\mathrm{S} 1$ group resulted in a more significant inhibitory effect on SW1990 cell growth in vivo, consistent with the results from the in vitro experiments $(\mathrm{p}<0.05)$. For example, the tumor volume in the nude mice on day 28 after the inoculation of SW1990 cells in the untransfected, Lv-Xnc, Lv-Snc and $\mathrm{Lv}-\mathrm{Xnc}+\mathrm{Snc}$ control groups and in the $\mathrm{Lv}-\mathrm{X} 1, \mathrm{Lv}-\mathrm{S} 1$ and $\mathrm{Lv}-\mathrm{X} 1+\mathrm{S} 1$ experimental groups was $1223.28 \pm 149.61$, $1173.45 \pm 176.14,984.29 \pm 216.42$ and $1089.03 \pm 51.11$, and $532.83 \pm 84.59,329.18 \pm 69.67$ and $232.58 \pm 88.08 \mathrm{~mm}^{3}$, respectively. The group with the smallest tumor volume was the $\mathrm{Lv}-\mathrm{X} 1+\mathrm{S} 1$ group. Tumor volume was smaller in the $\mathrm{Lv}-\mathrm{X} 1$ and $\mathrm{Lv}-\mathrm{S} 1$ groups than in all four control groups $(\mathrm{p}<0.05)$ (Fig. 4B).

Inhibition of XIAP and/or survivin slightly increased caspase-3/-7 activity and apoptosis. Through their binding to caspase-3/-7, XIAP and survivin play key roles in the apoptosis of cancer cells. To determine the effects of XIAP and survivin silencing on caspase activity and the apoptosis of SW1990 cells, the activity of caspase-3/-7 and apoptosis were examined in XIAP and/or survivin shRNA lentiviraltransfected SW1990 cells. In setting the level of caspase-3/-7 activity in the untransfected SW1990 cells at a value of 1, the 


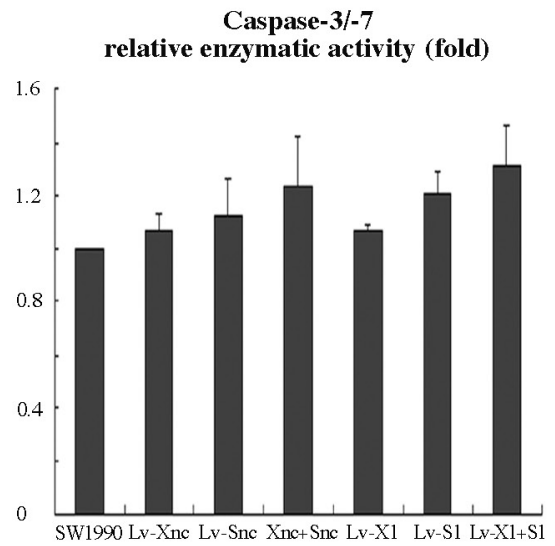

C
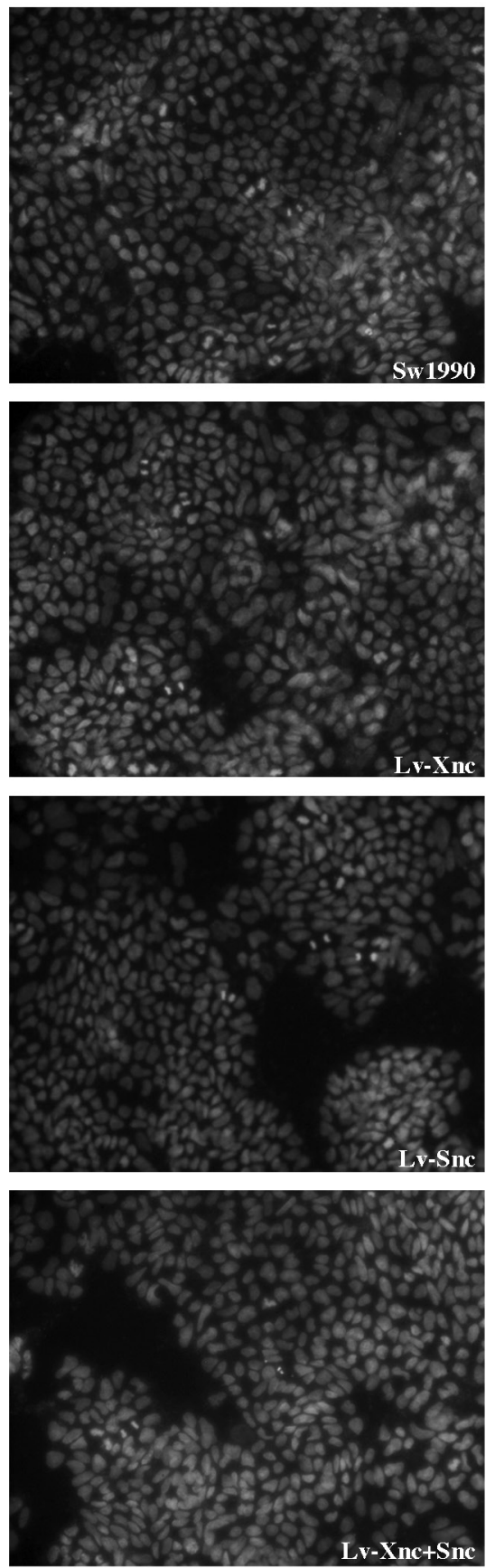

Apoptosis index (\%)
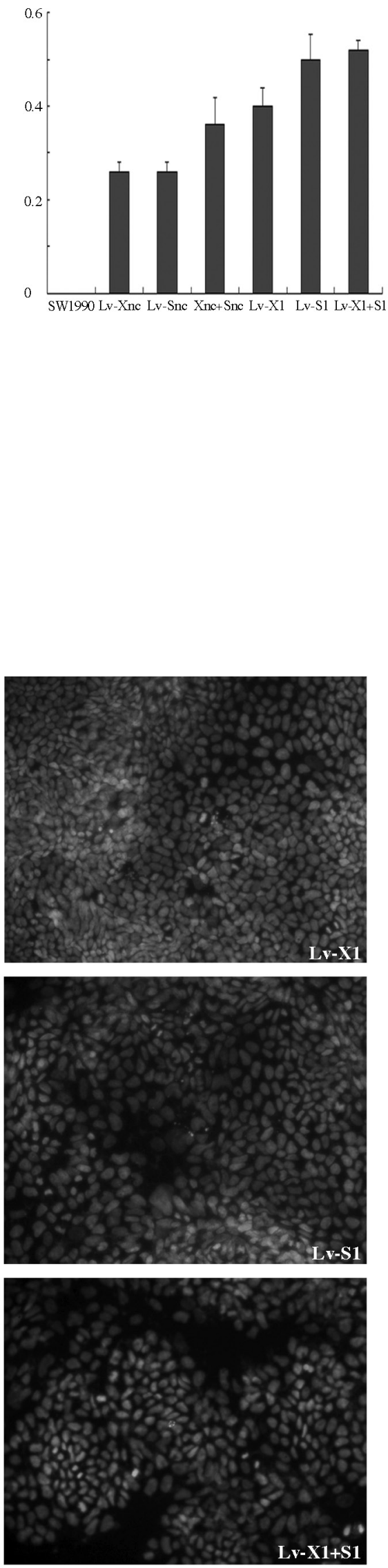

Figure 5. Effects of XIAP and/or survivin inhibition on (A) caspase-3/-7 activity and (B) apoptosis in SW1990 cells. Caspase-3/-7 activity levels in untransfected SW1990 cells were set at the value of 1, and the relative caspase-3/-7 activity of the other groups was calculated. (C) DAPI staining was used to examine SW1990 apoptosis, identified by the condensed, shrunken and ruptured nuclei. Apoptosis index $(\%)=$ apoptotic cells/total cells, observed and counted in the same microscopic field at a magnification of $\mathrm{x} 200$, selected from three random fields. 
relative enzymatic activity of caspase-3/-7 in the other three control groups ( $\mathrm{Lv}-\mathrm{Xnc}, \mathrm{Lv}-\mathrm{Snc}$ and $\mathrm{Lv}-\mathrm{Xnc}+\mathrm{Snc}$ ) and the three experimental groups (Lv-X1, Lv-S1 and $\mathrm{Lv}-\mathrm{X} 1+\mathrm{S} 1)$ was $1.07 \pm 0.06,1.12 \pm 0.14$ and $1.24 \pm 0.18$, and $1.07 \pm 0.02$, $1.21 \pm 0.08$ and $1.31 \pm 0.15$, respectively. Compared to the corresponding control groups, XIAP and survivin shRNA, either alone or in combination, slightly increased the caspase-3/-7 activity of the SW1990 cells. However, the differences in caspase-3/-7 activity among the control and experimental groups were not significant ( $\mathrm{p}>0.05)$ (Fig. 5A).

DAPI staining was employed to evaluate SW1990 apoptosis induced by XIAP and/or survivin suppression. Although no apoptosis was observed in the SW1990 cells without lentiviral transfection, the AI in the other three control groups (Lv-Xnc, Lv-Snc and $\mathrm{Lv}-\mathrm{Xnc}+\mathrm{Snc}$ ), and the three experimental groups (Lv-X1, Lv-S1 and $\mathrm{Lv}-\mathrm{X} 1+\mathrm{S} 1)$ was $0.13 \pm 0.01,0.13 \pm 0.01$ and $0.18 \pm 0.03$, and $0.2 \pm 0.02,0.27 \pm 0.03$ and $0.26 \pm 0.01$, respectively. The differences in the AI among the different groups were not statistically significant $(\mathrm{p}>0.05)$ (Fig. 5B). The AI measured by DAPI staining was consistent with the results of caspase-3/-7 activity.

\section{Discussion}

An underlying feature of pancreatic cancer is uncontrolled cell proliferation and impaired apoptosis (11). In human renal cancer cells, combined inhibition of XIAP and Bcl-2 has been shown to be capable of reversing apoptosis resistance in cancer cells (12). Triple inhibition of XIAP, Bcl-2 and Bcl-xl in bladder cancer cells has been shown to be even more effective in inducing cancer cell apoptosis (13). It has been reported that the combination of XIAP and tumor necrosis factor-related apoptosis-inducing ligand (TRAIL) is an effective approach to triggering apoptosis, despite Bcl-2 overexpression, and suppresses pancreatic cancer cell growth in vitro and in vivo (14). siRNAs against XIAP and survivin sensitize melanoma cells to apoptosis even more effectively than Bcl-2 (9). Therefore, it is possible that the simultaneous inhibition of IAP and the Bcl-2 axis in pancreatic cancer cells is not essential. As a therapeutic target, IAP is more to life than Bcl-2 (15). The Bcl-2 family consists of anti-apoptotic members, Bcl-2 and Bcl-xl, and pro-apoptotic members, Bax and $\mathrm{Bcl}-\mathrm{xs}$. In contrast to various other tumors, the expression of the prototypical pro-survival protein $\mathrm{Bcl}-2$ is normal or even decreased in pancreatic cancer (16), while Bcl-xl is known to be overexpressed (17). In this study, real-time PCR showed that Bcl-xl expression was higher than that of Bcl-2 in SW1990, Panc-1 and Mia-paca2 cells, using Bxpc-3 cells as a control (data not shown).

XIAP and survivin are two vital members of the IAP family; their baculoviral IAP repeat (BIR) motifs have been found to function primarily as inhibitors of apoptosis (18). The survivin-XIAP complex promotes increased XIAP stability to cell death stimulation against ubiquitination/ proteasomal destruction and synergistic inhibition of apoptosis via conserved BIR (19). XIAP and survivin are overexpressed in numerous human malignancies, and are known as poor prognostic factors (20). Furthermore, it has been reported that intermolecular cooperation between XIAP and survivin stimulates tumor cell invasion and promotes metastasis (8); thus,
XIAP and survivin are also direct metastasis genes, and their inhibition may be used in anti-metastasis therapy in patients with cancer (in particular advanced cancer) and metastatic diseases, such as pancreatic cancer.

In this study, we found that lentivirus-based vectors were extremely efficient and persisted in transducing pancreatic cancer cells (Figs. 2 and 3). To determine whether lentiviral-mediated shRNA was capable of stably inhibiting the expression of XIAP and survivin, pooled clones were expanded and stably transfected cells were lysed for Western blot analysis 1 month later. The results showed that the expression of XIAP and survivin in the Lv-X1 and Lv-S1 cells was still markedly reduced (Fig. 3). Consistent with previous studies (21), we did not find an association between the downregulation of XIAP or survivin and a significantly increased spontaneous apoptosis rate, based not only on the levels of caspase-3/-7 activity (Fig. 5A), but also on the apoptosis index (Fig. 5B) as determined by DAPI staining. To validate the specificity of RNAi targeting XIAP and to avoid off-target phenomena, the cells were transfected with lentivirus carrying three different shRNA sequences against XIAP or survivin, and the level of another IAP family protein, survivin or XIAP, respectively, was also determined. The results showed that survivin was not affected by the knockdown of XIAP, and vice versa (Figs. 2 and 3). Of note, we observed that when the expression of XIAP was down-regulated, the expression of survivin was up-regulated, and vice versa. It may be that XIAP and survivin are functionally linked. It has been reported that HIV-1 infection mediates the up-regulation of IAP via HIV-1 Vpr (22). The lentivirus vector used was replication-deficient $\mathrm{HIV}$, which may explain this phenomenon.

In this study, we determined whether cells stably transfected with lentivirus decreased long-term survival and proliferation in vitro and in vivo without intervention. The cell growth curves and results from a xenograft model showed that the growth of cells transfected with Lv-X1+Lv-S1 was significantly inhibited: growth curves were low and flat (Fig. 4A), and the BALB/c nude mice inoculated with $\mathrm{Lv}-\mathrm{X} 1+\mathrm{S} 1$ cells developed the smallest tumors compared to the control (Fig. 4B). Notably, compared to the inhibition of XIAP via $\mathrm{Lv}-\mathrm{X} 1$, inhibition of survivin via Lv-S1 was more efficient in enhancing apoptosis (Fig. 5A and B) and suppressing cell proliferation in vitro and in vivo (Fig. 4). It is possible that survivin is more important than XIAP in the apoptotic pathways of pancreatic cancer SW1990 cells. It has been reported that XIAP inhibits cell proliferation via the down-regulation of cyclins A and D1 and the induction of the cyclin-dependent kinase inhibitors $\mathrm{p} 21^{\mathrm{Cip} 1 / \mathrm{Waf} 1}$ and $\mathrm{p} 27^{\mathrm{Kip} 1}$ in human endothelial cells (23). In this study, we found that the inhibition of XIAP suppressed pancreatic cancer cell proliferation. One possible mechanism of this effect is that caspase activity is necessary for normal cell cycle progression. High caspase activity in many malignant tumors, for example pancreatic cancer, results in increased cell proliferation. Survivin not only inhibits caspase-dependent apoptosis, caspase-independent cell death, and mitochondrial and TRAIL-mediated apoptosis, it also controls mitosis, chromosomal attachment and the spindle-assembly checkpoint (24). Therefore, in terms of cell proliferation, the inhibition of survivin was more effective than that of XIAP. 
Functional cross-talk has been reported between the PI3K/ Akt survival pathway and the NF-KB apoptotic pathway in pancreatic cancer via XIAP and survivin expression (25). We observed that XIAP and/or survivin knockdown in the absence of additional treatment significantly decreased long-term survival and proliferation in vitro and in vivo, but did not lead to a detectable increase in apoptosis. This effect may be associated with the attenuation of the PI3K/Akt cell survival pathway and the NF- $\mathrm{KB}$ signal pathway. For example, XIAP induces NF- $\kappa B$ activation via a BIR1/TAB1 interaction and BIR1 dimerization (26), and fibronection protects cancer cells from TNF- $\alpha$-induced apoptosis via the Akt/survivin pathway (27). It is known that the differential splicing of survivin pre-mRNA results in three new survivin variants with different anti- or pro-apoptotic properties: survivin $\alpha$, survivin-2B and survivin $\Delta \mathrm{Ex} 3$ (28). Survivin-2B is thought to be pro-apoptotic and has been reported to contribute to drug resistance. However, in this study no correlation supporting such a role was found. Additionally, no clear positive correlation for the anti-apoptotic isoforms survivin $\alpha$ and survivin $\Delta \mathrm{Ex} 3$ was observed (data not shown). Therefore, the inhibition of survivin may be insufficient and the expression of the isoforms may be altered, even more than they are functionally linked. This point requires further study, and this mechanism suggests that the inhibition of XIAP and survivin expression alone results in the suppression of pancreatic cancer cell growth in vitro and in vivo, without additional stimulation.

In conclusion, our findings demonstrate for the first time that the suppression of XIAP and survivin expression via lentivirus-mediated shRNA may serve as a novel therapeutic strategy for pancreatic cancer, and warrants the further investigation of lentivirus transfection in cancer gene therapy studies.

\section{Acknowledgements}

This study was supported by a grant from the National Natural Science Foundation of China (no. 30872492) and a grant from the Natural Science Foundation of Hunan Province (no. 088JJ3042).

\section{References}

1. Jemal A, Siegel R, Ward E, Hao YP, Xu JQ and Thun MJ: Cancer Statistics, 2009. CA Cancer J Clin 59: 225-249, 2009.

2. Evan GI and Vousden KH: Proliferation, cell cycle and apoptosis in cancer. Nature 411: 342-348, 2001.

3. Wang SL, Hawkins CJ, Yoo SJ, Muller HAJ and Hay BA: The Drosophila caspase inhibitor DIAP1 is essential for cell survival and is negatively regulated by HID. Cell 98: 453-463, 1999.

4. Zangemeister-Wittke U and Simon HU: An IAP in action - the multiple roles of survivin in differentiation, immunity and malignancy. Cell Cycle 3: 1121-1123, 2004.

5. Kumamoto $\mathrm{H}$ and Ooya $\mathrm{K}$ : Expression of survivin and $\mathrm{X}$ chromosome-linked inhibitor of apoptosis protein in ameloblastomas. Virchows Archiv 444: 164-170, 2004.

6. LaCasse EC, Mahoney DJ, Cheung HH, Plenchette S, Baird S and Korneluk RG: IAP-targeted therapies for cancer. Oncogene 27: 6252-6275, 2008.

7. Chen P, Li J, Ge LP, Dai CH and Li XQ: Prognostic value of survivin, X-linked inhibitor of apoptosis protein and second mitochondria-derived activator of caspases expression in advanced non-small-cell lung cancer patients. Respirology 15: 501-509, 2010.

8. Mehrotra S, Languino LR, Raskett CM, Mercurio AM, Dohi T and Altieri DC: IAP regulation of metastasis. Cancer Cell 17: 53-64, 2010
9. Chawla-Sarkar M, Bae SI, Reu FJ, Jacobs BS, Lindner DJ and Borden EC: Downregulation of Bcl-2, FLIP or IAPs (XIAP and survivin) by siRNAs sensitizes resistant melanoma cells to Apo2L/TRAIL-induced apoptosis. Cell Death Differ 11: 915-923, 2004

10. Kyriazis AP, McCombs WB III, Sandberg AA, Kyriazis AA, Sloane NH and Lepera R: Establishment and characterization of human pancreatic adenocarcinoma cell line SW-1990 in tissue culture and the nude mouse. Cancer Res 43: 4393-4401, 1983.

11. Hanahan D and Weinberg RA: The hallmarks of cancer. Cell 100: 57-70, 2000.

12. Bilim V, Yuuki $K$, Itoi $T$, Muto $A$, Kato $T$, Nagaoka $A$, Motoyama $\mathrm{T}$ and Tomita $\mathrm{Y}$ : Double inhibition of XIAP and $\mathrm{Bcl}-2$ axis is beneficial for retrieving sensitivity of renal cell cancer to apoptosis. Br J Cancer 98: 941-949, 2008.

13. Kunze D, Wuttig D, Fuessel S, Kraemer K, Kotzsch M, Meye A, Grimm MO, Hakenberg OW and Wirth MP: Multitarget siRNA inhibition of antiapoptotic genes (XIAP, BCL2, BCL-X-L) in bladder cancer cells. Anticancer Res 28: 2259-2263, 2008.

14. Vogler M, Walczak H, Stadel D, Haas TL, Genze F, Jovanovic M, Gschwend JE, Simmet T, Debatin KM and Fulda S: Targeting XIAP bypasses Bcl-2-mediated resistance to TRAIL and cooperates with TRAIL to suppress pancreatic cancer growth in vitro and in vivo. Cancer Res 68: 7956-7965, 2008.

15. Liston P, Fong WG and Korneluk RG: The inhibitors of apoptosis: there is more to life than Bcl2. Oncogene 22: 8568-8580, 2003.

16. Evans JD, Cornford PA, Dodson A, Greenhalf W, Foster CS and Neoptolemos JP: Detailed tissue expression of bcl-2, bax, bak and bcl-x in the normal human pancreas and in chronic pancreatitis, ampullary and pancreatic ductal adenocarcinomas. Pancreatology 1: 254-262, 2001.

17. Hinz S, Trauzold A, Boenicke L, Sandberg C, Beckmann S, Bayer E, Walczak H, Kalthoff $\mathrm{H}$ and Ungefroren $\mathrm{H}$ : Bcl-XL protects pancreatic adenocarcinoma cells against CD95- and TRAILreceptor-mediated apoptosis. Oncogene 19: 5477-5486, 2000.

18. Srinivasula SM and Ashwell JD: IAPs: what's in a name? Mol Cell 30: 123-135, 2008.

19. Dohi T, Okada K, Xia F, Wilford CE, Samuel T, Welsh K, Marusawa H, Zou H, Armstrong R, Matsuzawa S, Salvesen GS, Reed JC and Altieri DC: An IAP-IAP complex inhibits apoptosis. J Biol Chem 279: 34087-34090, 2004.

20. Krepela E, Dankova P, Moravcikova E, Krepelova A, Prochazka J, Cermak J, Schutzner J, Zatloukal P and Benkova K: Increased expression of inhibitor of apoptosis proteins, survivin and XIAP, in non-small cell lung carcinoma. Int J Oncol 35: 1449-1462, 2009.

21. Sensintaffar J, Scott FL, Peach R and Hager JH: XIAP is not required for human tumor cell survival in the absence of an exogenous death signal. BMC Cancer 10: 11, 2010.

22. Zhu Y, Roshal M, Li F, Blackett J, Planelles V: Upregulation of survivin by HIV-1 Vpr. Apoptosis 8: 71-79, 2003.

23. Levkau B, Garton KJ, Ferri N, Kloke K, Nofer JR, Baba HA, Raines EW and Breithardt G: xIAP induces cell-cycle arrest and activates nuclear factor-kappaB: new survival pathways disabled by caspase-mediated cleavage during apoptosis of human endothelial cells. Circ Res 88: 282-290, 2001.

24. Altieri DC: survivin, cancer networks and pathway-directed drug discovery. Nat Rev Cancer 8: 61-70, 2008.

25. Hamacher R, Schmid RM, Saur D and Schneider G: Apoptotic pathways in pancreatic ductal adenocarcinoma. Mol Cancer 7: 64, 2008.

26. Lu M, Lin SC, Huang YH, Kang YJ, Rich R, Lo YC, Myszka D, Han JH and Wu H: XIAP induces NF-kappa B activation via the BIR1/TAB1 interaction and BIR1 dimerization. Mol Cell 26: 689-702, 2007

27. Fornaro M, Plescia J, Chheang S, Tallini G, Zhu YM, King M, Altieri DC and Languino LR: Fibronectin protects prostate cancer cells from tumor necrosis factor-alpha-induced apoptosis via the AKT/survivin pathway. J Biol Chem 278: 50402-50411, 2003.

28. Caldas H, Jiang YY, Holloway MP, Fangusaro J, Mahotka C, Conway EM and Altura RA: survivin splice variants regulate the balance between proliferation and cell death. Oncogene 24: 1994-2007, 2005. 